\title{
Channel switch rejuvenates neurons
}

DOI:

10.1038/nrd2387
Why the dopaminergic neurons of the brain's substantia nigra pars compacta $(\mathrm{SNc})$ region degenerate and die in Parkinson's disease (PD) is not well understood. Chan and colleagues, writing in Nature, now show that the reliance of these neurons on a particular class of $\mathrm{Ca}^{2+}$ channels for driving their basal activity, known as pacemaking, could be an important factor. By blocking these channels in an animal model of PD - and thereby inducing adult neurons to switch to other channels that are used by juvenile neurons for pacemaking - the neurons can be protected from disease progression. This 'rejuvenation' could therefore represent a new therapeutic strategy for the disease.

The motor symptoms that are characteristic of PD are associated with the degeneration and death of adult SNc dopaminergic neurons. The authors determined that these neurons express $\mathrm{Ca}_{\mathrm{v}} 1.3 \mathrm{Ca}^{2+}$ channels rather than the more common $\mathrm{Ca}_{\mathrm{v}} 1.2 \mathrm{Ca}^{2+}$ channels. To investigate the importance of this, they studied $C a_{v} 1.3^{-/-}$mice and observed that the SNc dopaminergic neurons had normal pacemaking using $\mathrm{Na}^{+} /$hyperpolarization-activated and cyclic nucleotide-gated cation (HCN) channels.

Next, Chan and colleagues demonstrated that $\mathrm{HCN}$ channels are important for the pacemaking of SNc dopaminergic neurons in juvenile wild-type mice, but the dependence on these channels decreases with age, corresponding with an increased dependence on $\mathrm{Ca}_{\mathrm{v}} 1.3 \mathrm{Ca}^{2+}$ channels. The authors investigated the known sensitivity of HCN channels to cyclic adenosine monophosphate (cAMP), which is dampened by $\mathrm{Ca}^{2+}$, and observed that suppressing adenylyl cyclase, the enzyme that produces cAMP, normalizes the $\mathrm{HCN}$ channel gating in $\mathrm{Ca} 1.3^{-/-}$neurons, suggesting that the strategy used by juvenile SNc dopaminergic neurons could be restored.

So, to determine if adult $\mathrm{SNc}$ dopaminergic neurons could be rejuvenated in vivo, wild-type mice were given the $\mathrm{Ca}^{2+}$-channel blocker isradipine. Pacemaking activity of SNc dopaminergic neurons from these mice was insensitive to isradipine compared with non-treated controls, indicating a switch in the channels used for pacemaking.

Hypothesizing that rejuvenation might protect SNc dopaminergic neurons from degeneration, the authors then assessed the effects of isradipine in an experimental model of PD created by injection of the toxin MPTP. After a 5-week period of MPTP injections, loss of SNc dopaminergic neurons was reduced by nearly half in mice treated with isradipine, indicating that rejuvenation confers protection against $\mathrm{PD}$ progression. As isradipine is already widely used as a treatment for hypertension and stroke, the potential of this approach to ameliorate PD in humans could soon be evaluated in clinical trials.

Bethan Hughes

ORIGINAL RESEARCH PAPER Chan, C. S. et al. 'Rejuvenation' protects neurons in mouse models of Parkinson's disease. Nature 447, 1081-1086 (2007)

FURTHER READING Schapira, A. H. V. et al. Novel pharmacological targets for the treatment of Parkinson's disease. Nature Rev. Drug Discov. 5 845-854 (2006) 